\title{
Internalised weight stigma as a mediator of the relationship between experienced/perceived weight stigma and biopsychosocial outcomes: a systematic review
}

\author{
Hugh Bidstrup $\mathbb{D}^{1}$, Leah Brennan ${ }^{2}$, Leah Kaufmann ${ }^{1}$ and Xochitl de la Piedad Garcia $\mathbb{D}^{1 凶}$ \\ (c) The Author(s), under exclusive licence to Springer Nature Limited 2021
}

OBJECTIVE: To systematically review studies that have assessed the mediating role of internalised weight stigma on the relationship between experienced/perceived weight stigma and any biopsychosocial outcomes.

METHODS: PsycINFO, PsycExtra, Web of Science, CINAHL, Medline and Embase were systematically searched. Identified studies were double screened (HB and XPG).

RESULTS: Seventeen studies (across 16 articles) met our inclusion criteria $(N=21,172)$, and almost all studies measured only psychological outcomes $(n=15)$. Eight studies found consistent evidence for internalised weight stigma as a mediator of the relationship between experienced/perceived weight stigma and disordered eating outcomes. Preliminary evidence was found for the mediating role of internalised weight stigma on the relationship between experienced/perceived weight stigma and body shame, body dissatisfaction, exercise behaviour, healthcare experiences and behaviours, bodily pain and parental weight talk. However, the findings were inconsistent for depression and anxiety, although only two studies reported these.

CONCLUSION: This review provides preliminary evidence for internalised weight stigma as an intervening variable in the relationship between experienced/perceived weight stigma and adverse health outcomes. Results suggest that there are potential benefits of interventions addressing internalised weight stigma to improve health outcomes. However, these findings must be considered in the context of the psychometric limitations of the Weight Bias Internalisation Scale, which was used in all but one study.

International Journal of Obesity (2022) 46:1-9; https://doi.org/10.1038/s41366-021-00982-4

\section{INTRODUCTION}

Many of the negative health outcomes associated with overweight/obesity are also associated with individuals' experiences, perceptions, anticipation and internalisation of weight stigma $[1,2]$. Weight stigma broadly refers to the social penalising or denigration of individuals because of their weight [3, 4]. Typically, weight stigma manifests as stereotypical beliefs (e.g., lacking willpower), negative attitudes (e.g., "I do not like people with overweight") or behaviours (e.g., social rejection, discrimination). Perceptions and experiences of weight stigma by people with overweight/obesity can range from seeing negative representations of individuals with higher weight in the media (e.g., "fat Monica" in the popular situation comedy Friends), to being called names, to being treated differently because of one's weight (e.g., less likely to be interviewed despite equal qualifications and experience compared to their non-overweight counterparts [5]). Research shows these experiences and perceptions of stigma are prevalent across settings, such as in the home, in the workplace, and in healthcare $[4,6]$.
The literature on weight stigma often makes the distinction between felt and enacted stigma (i.e., perceived versus experienced weight stigma). However, some measures that purport to measure one type of stigma include items that seem to measure the other type of stigma. For example, there are some items on the widely used Stigmatising Situations Inventory [7], which intends to measure experienced weight stigma, that could arguably be measuring perceived weight stigma (e.g., "being stared at in public") (p. 224). Further, we would argue that from the perspective of the target, whether people actually stared at them or the person just felt they did might make no difference. Similarly, Emmer et al. [1] meta-analysed the correlates of experienced and perceived weight stigma and classified these types of stigma under the umbrella term 'public stigma'. In this review, we have chosen not to make a distinction between perceived/experienced weight stigma.

Recent research [8-10] has found that perceived/experienced weight stigma is linked to poor physical and mental health outcomes. For example, cross-sectional studies show weight

${ }^{1}$ School of Behavioural and Health Sciences, Australian Catholic University, Melbourne 3065, Australia. ${ }^{2}$ School of Psychology and Public Health, La Trobe University, AlburyWodonga 3690, Australia. ${ }^{凶}$ email: xochitl.delapiedadgarcia@acu.edu.au

Received: 14 July 2021 Revised: 14 September 2021 Accepted: 29 September 2021

Published online: 9 October 2021 


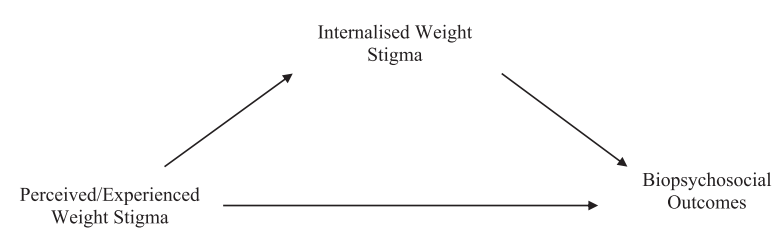

Fig. 1 Internalised weight stigma mediates weight stigma effects. This model proposes that internalised weight stigma mediates the relationship between weight stigma and poor health outcomes. Specifically, that perceived/experienced weight stigma is positively associated with internalised weight stigma, which is in turn associated with more adverse biopsychosocial outcomes (e.g., higher rates of disordered eating, lower levels of body image satisfaction).

stigma is associated with anxiety, substance use, antisocial behaviour [11], depressive symptoms, low self-esteem, diabetes risk, and social isolation [12,13], even when controlling for BMI. Intriguingly, based on "scattered evidence" [14] (p. 791), some argue that stigmatising individuals with overweight/obesity can, for some, be a trigger to seek help for weight loss [15]. However, there is extensive evidence to the contrary. Specifically, even when controlling for BMl, experiencing weight stigmatisation is consistently associated with adverse health outcomes, such as increased mortality risk [16] and poorer mental and physical health $[1,9,17,18]$. In addition, weight stigma is associated with unhealthy eating and weight control behaviours (e.g., binge eating and emotional overeating $[19,20])$. Experimental evidence also shows that individuals exposed to weight stigmatising stimuli (e.g., videos or news articles) had higher food intake than those not exposed to weight stigmatising material [21, 22].

Longitudinal evidence from a sample of 3609 adults in the United Kingdom indicated that from baseline to a 4-year followup, perceived weight stigma accounted for $27 \%$ of the prospective association between overweight/obesity and greater physiological dysregulation (as measured by several biomarkers of metabolic, cardiovascular, and immune functioning) [23]. This effect remained even when adjusted for BMI, initial health conditions, physiological dysregulation levels, sociodemographic characteristics, and health conditions. Similarly, a recent meta-analysis [1] found that high perceived/experienced weight stigma is associated with poorer mental health outcomes $(r=-0.33, p<0.001$, $k=241$ ). Lastly, there is preliminary evidence that anticipated weight stigma predicts disordered eating $[2,24]$, unhealthy weight control behaviour (e.g., fasting, purging) [25], alcohol use, sleep disturbances, and comfort eating (while controlling for BMI) [24]. In sum, research has found that weight stigma (perceived, experienced and anticipated) is an important predictor of several negative health outcomes that are often attributed primarily to overweight/obesity [26].

\section{Internalisation of weight stigma}

In addition to perceived, experienced, and anticipated weight stigma, researchers have increasingly become interested in the extent to which individuals internalise weight stigma (also referred to as self-stigma or self-directed weight stigma). It has been proposed [27] that internalised weight stigma is characterised by several components of an individual's perception of the self and their identification as having overweight/obesity. These include (a) awareness of and agreement with negative stereotypes about weight (e.g., "Fat people are lazy"), (b) application of negative stereotypes to the self (e.g., "I am lazy"), and (c) mistreatment or devaluation of oneself because of perceived self-classification as 'overweight' (e.g., "I am less attractive than most other people because of my weight") [27]. Meta-analytic evidence shows that internalised weight stigma is associated with poor mental health outcomes (e.g., depression, body image disturbance; $r=-0.39, p$ $<0.001, k=222)$ [1].

In the last decade, there has been increased research attention devoted to investigating the role of internalised weight stigma as a mediator of the relationship between perceived/experienced weight stigma and a range of biopsychosocial outcomes (see Fig. 1 below). For instance, a theoretical model by Tylka et al. [28] suggests that internalised weight stigma mediates the relationship between experienced/perceived weight stigma and psychological well-being and physical health.

There is preliminary evidence to support this theoretical model $[29,30]$. Specifically, Forbes and Donovan [31] found that internalised weight stigma mediated the relationship between experiences of weight stigma and body shame in a sample of Australian women with overweight/obesity. Specifically, they found that more frequent experiences of weight stigma were associated with higher levels of internalised weight stigma, which in turn were related to higher levels of body shame (after controlling for BMI). In addition, in a sample of 631 undergraduate students, O'Brien et al. [32] found that internalised weight stigma mediated the relationship between experiences of weight stigma and emotional eating and uncontrolled eating (controlling for weight status, age and gender). Interestingly, there are other reports of the mediating role of internalised weight stigma on the relationship between experienced weight stigma and a number of outcomes, such as depression and anxiety [33], bodily pain [29], and exercise behaviour [34]. These studies all shower higher experienced/perceived weight stigma is associated with higher internalised weight stigma, which is in turn associated with poorer outcomes (e.g., higher levels of depression, anxiety, bodily pain and lower levels of exercise behaviour).

\section{The current review}

There is a small but growing body of evidence assessing internalised weight stigma as a mediator of the relationship between perceived/experienced weight stigma and a range of biopsychosocial outcomes. A review of the existing literature is timely as it will provide (a) a synthesis of the current evidence, and (b) an opportunity to identify future directions both with regards to research questions and methodological approaches in the area. Thus, the aim of the current study is to conduct a systematic literature review of studies measuring the mediating role of internalised weight stigma in the relationship between perceived/ experienced weight stigma and biopsychosocial outcomes.

\section{METHODS}

Preferred Reporting Items for Systematic reviews and MetaAnalyses (PRISMA) [35] statement was used for this review and rated according to Assessing the Methodological Quality of Systematic Reviews (i.e., AMSTAR; see Table S1) [36]. Our review methods were established prior to data extraction and were preregistered in PROSPERO (ID: CRD42020205823).

\section{Search strategy}

The literature search was conducted in August 2020 (and updated in June 2021) via six databases: PsycINFO, PsycExtra, Web of Science Core Collection, CINAHL, Medline Complete, and Embase. In addition, grey literature was included in the searches conducted in the first three databases listed above. Search terms were based on two concepts. Concept 1 included terms that refer to all weight stigma domains identified in the literature (i.e., experienced, perceived, internalised, or self-directed weight stigma). Concept 2 included any terms related to mediation analysis (e.g., mediation, mediator, SEM, path analysis). The terms corresponding to these concepts were searched for in title and abstracts. In addition to 
this, relevant subject terms were selected for each database, when applicable/available (see Table S2 for search terms).

\section{Inclusion criteria}

We included any study that reported data on at least one sample of adults (ages $\geq 18$ years), with no restrictions on sample type (e.g., community, clinical) or BMI. To be included, studies had to include measures of (a) a form of perceived/experienced weight stigma as a predictor variable, (b) internalised weight stigma as a mediator, and (c) any biological, psychological, or social outcome measure. Theses, dissertations, conference presentations/ abstracts, as well as published studies were all included, provided they reported enough data for inclusion (HB and XPG emailed a request to all authors if they did not report sufficient information/ data. We included unpublished studies because prior to conducting our review, we aimed to assess for publication bias). However, book chapters/sections were excluded from this review, as well as studies not written in English. We had no limit on the year of publication.

\section{Screening and data extraction}

Studies were double screened by $\mathrm{HB}$ and XPG, using the application Rayyan [37] at both the title and abstract, and fulltext screening stages. Conflicts were resolved by discussion. HB extracted the following information from each study, when reported: country, sample characteristics (e.g., sample size, recruitment techniques/source, frequencies or descriptive statistics on gender, age, BMI), measure(s) used (for the predictor, mediator and outcomes variables), type of mediation (e.g., structural equation model), as well as inferential statistics for the mediation (total, direct and indirect effect(s) $\left[c, c^{\prime}\right.$, and $\left.a^{*} b\right]$, and any measure of error [i.e., $95 \% \mathrm{Cls}$ and/or standard error]).

\section{Quality assessment}

To assess the quality of the studies included in the review, we used guidelines for reporting mediational analysis [38]. These guidelines were intended for evaluation of the methodological quality of mediation studies both in intervention(s) and randomised controlled trials, and in observational studies. However, due to the cross-sectional nature of the data in the current review, we only included questions relevant to observational studies (see Table S3). HB completed the quality assessment.

\section{RESULTS}

Figure 2 displays the PRISMA flow-chart. Our search returned 1379 articles. After removing duplicates, this number was reduced to 806 , of which 768 were excluded after title and abstract screening. When we applied our exclusion criteria to the remaining 38 fulltext articles, 22 were excluded (reasons for exclusion are listed in Fig. 2 and a full list of these excluded studies are provided in Table S4). Sixteen articles met our inclusion criteria for this review. Of these, one reported data across two studies (i.e. two independent samples), leaving us with a total of 17 studies for extraction.

\section{Study characteristics}

Table S5 shows an overview of the study characteristics for each of the included studies. Data for 17 studies was extracted $(N=$ 21,172). These studies collected data in seven different countries: United States $(n=11)$, Australia $(n=3)$, Germany $(n=3)$, Canada $(n=2)$, France $(n=1)$, Spain $(n=1)$, and the United Kingdom $(n=1)$ (Note that one study had data from six different countries). Four studies sampled women only; all but two studies had a higher ratio of women to men (average $\%$ of women $=73 \%$, range $35-97 \%$ ) (Note to describe gender ratios in the included studies, we used the percentage of women in each sample. This is because all studies in this review only reported that participants identified as either men/male or women/female. As many individuals do not identify as any of these, we encourage future researchers to allow participants to identify as other options (e.g., "intersex", "not specified", "non-binary", or "prefer not to say")).

There were 15 published studies, and 2 unpublished studies (one thesis and one dissertation). Participants in these studies were sampled from the community $(n=8)$, university student cohorts $(n=5)$ and from clinical $(n=2)$ populations. Two studies had a combination of these sample types-a combined student and community $(n=1)$, and a student and clinical sample $(n=1)$. Almost all studies assessed the mediating role of internalised weight stigma on the relationship between perceived/experienced weight stigma and psychological outcomes $(n=15)$. Only two studies assessed a biological $(n=1)$ or a social $(n=1)$ outcome. Almost half of the studies used structural equation modelling for their mediation analysis $(n=8)$. The remaining studies used serial/parallel mediation, or a bootstrapped analysis through PROCESS [39] $(n=8)$. One study did not specify the type of mediation used, only the statistical package (i.e., MPlus) [40].

\section{Perceived/experienced weight stigma measures}

There were several measures of perceived/experienced weight stigma used across studies. Six studies [29, 31, 41-44] used the Stigmatizing Situations Inventory [7] (or the brief/modified version of this scale). Three studies [30,32, 45] used either the original or modified version (or the original translated to German) of the Perception of Teasing Scale [46]. Five studies used a range of different, psychometrically validated measures [33, 44, 47-49]. The three remaining studies used ad-hoc measures, and of these, one reported good internal consistency [34], and the other two only had two [50] and three items [40], respectively.

\section{Internalised weight stigma measures}

Sixteen of the 17 studies in this review used a form of the Weight Bias Internalisation Scale [27]. This included the original scale ( $n=$ 4 ), the modified version (which uses a 4-point instead of a 10point Likert scale; $n=9$ ) [51], or the original version translated to German $(n=1)$ [30]. One of the 17 studies used the Weight Bias Internalisation Scale in its original form but also used it as a bidimensional measure, having obtained two factors in a bi-factor analysis of its items [41]. Lastly, one study [33] used the Weight Self-Stigma Questionnaire [52].

\section{Quality assessment}

Every study in this review was assessed using the criteria of the quality assessment (see Table S3 for assessment items, and Table S6 for quality assessment results). Most studies used measures that have good to excellent internal consistency. In addition, all studies ( $n=17)$ specified their main effect(s) of interest (indirect and/or direct effect), and almost all studies specified what statistical test would assess their mediation, including references $(n=15)$. Notably, only one published study in this review reported an a-priori power analysis [41].

The main issue with the quality of reporting in the studies had to be with reporting of statistical information. Most studies included descriptive statistics for each variable and all studies included a measure of effect for both the predictor-mediator (path a) and mediator-outcome paths (path $b ; n=17$ ). However, many studies-mostly structural equation models-reported ' $a$ ' and ' $b$ ' mediation paths, without any estimate of error for these paths $(n=11)$. In addition, many studies did not report an effect size estimate, with the corresponding error estimate, for the indirect effect(s) $\left(a^{*} b ; n=6\right)$. Instead, they either indicated that (a) the indirect effect was significant (or not), or (b) mediation was present/absent while providing incomplete statistical information (e.g., reporting a direct and/or total effect coefficient, often without an estimate of error/precision). Lastly, many studies did 


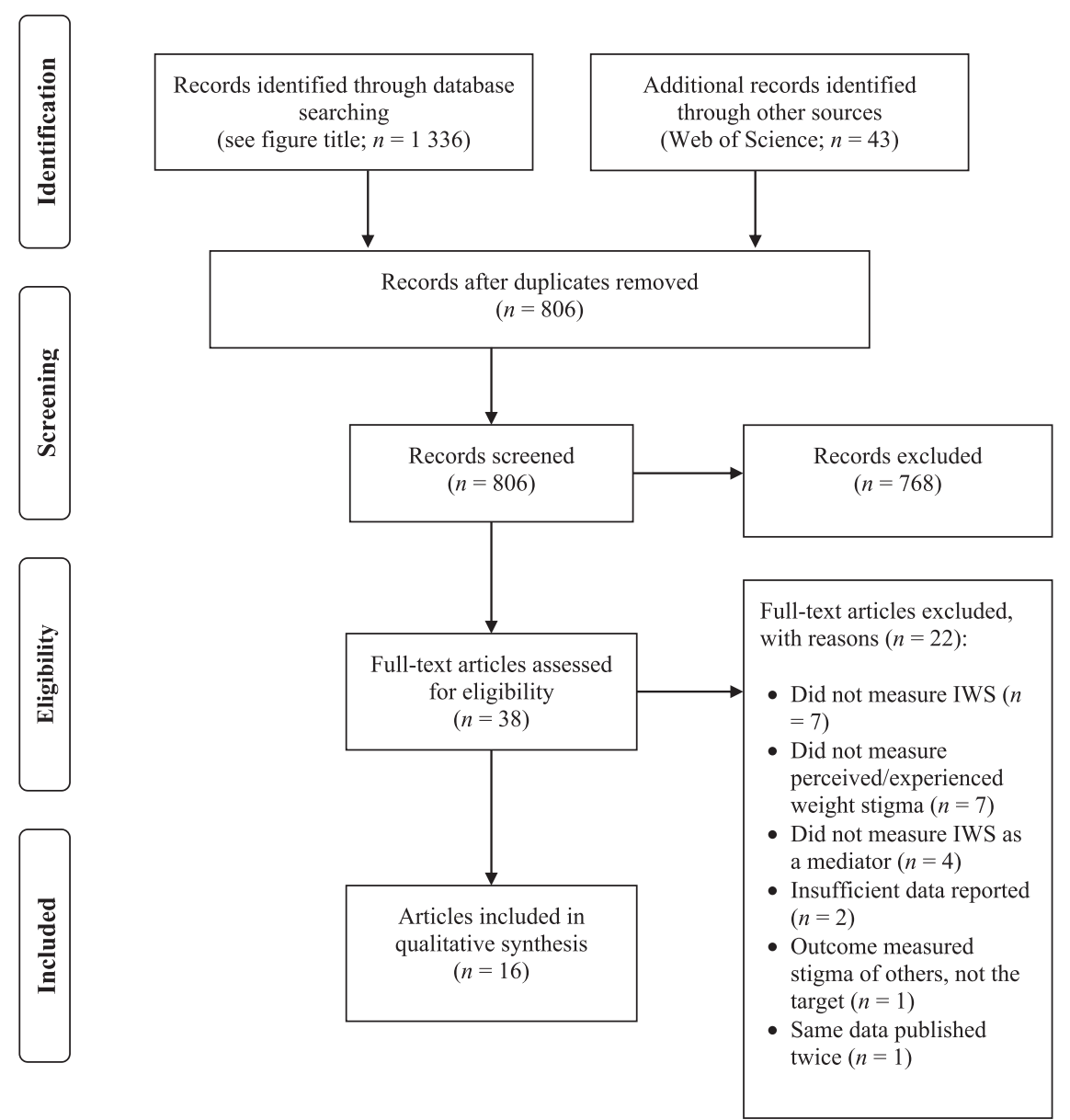

Fig. 2 PRISMA flowchart showing the study selection process. Flowchart for the current systematic review. Databases searched: CINAHL, Embase, Medline, PsycEXTRA, and PsycINFO.

not report standardised effect sizes for their indirect effects; thus the effect sizes were neither comparable nor assessable in terms of relative magnitude.

\section{Synthesis of results}

Studies in the current review $(n=17)$ evaluated internalised weight stigma as a mediator of the relationship between perceived/experienced weight stigma and a variety of biopsychosocial outcomes $(k=18)$. Specifically, studies assessed whether internalised weight stigma mediated the relationship between experiences of weight stigma and the following outcomes: (a) disordered eating $(k=8)$, (b) depression and/or anxiety $(k=2)$, (c) a combination of depression and disordered eating $(k=2)$, (d) other psychological outcomes ( $k=4$ : body shame, body dissatisfaction, exercise behaviour, and healthcare experiences and behaviours, respectively), and (e) biological and social outcomes ( $k=2$ : bodily pain and parental weight talk). The findings of these studies will be presented under subheading classifications as outlined above. All indirect effects except for two, whether statistically significant or not, were in the expected direction. That is, higher experienced/perceived weight stigma was positively related with higher internalised weight stigma, which in turn was positively related with more adverse outcomes (e.g., higher levels of body shame and disordered eating, and lower levels of exercise). The two exceptions include one article that found that internalised weight stigma was negatively related to anxiety (albeit non-significantly) [43], and the other article found that internalised weight stigma was negatively related to dietary restriction (in one study this mediation was significant, but nonsignificant in the other study) [44].

\section{Psychological outcomes}

Disordered eating: Eight studies measured disordered eating outcomes (estimated effect sizes are listed in Table S5). The consistent finding across studies was that internalised weight stigma significantly mediated the relationship between perceived/experienced weight stigma and disordered eating outcomes. Specifically, internalised weight stigma mediated the relationship between perceived/ experienced weight stigma and eating disturbance (eating-related pathology and emotional eating) [49], eating behaviour outcomes (binge eating, dietary restraint and disinhibition) [41], emotional and uncontrolled eating [32, 47], loss of control over eating [32], disordered eating (in adolescents and young adults) [30], dietary restraint (in non-clinical, primary school-aged students) [45] and purging [44]. Interestingly, Romano et al. [44] had additional but somewhat mixed findings across two samples for disordered eating. When experienced weight stigma was measured by the EDS, internalised weight stigma was a mediator of the relationship between experienced weight stigma and binge eating but not dietary restriction; and when experienced weight stigma was measured by the SSI, internalised weight stigma was a mediator for dietary restriction but not binge eating [44].

Importantly, one study [41] included in this review did a bifactor analysis (A bi-factor analysis is a form of confirmatory factor analysis that allows for the variance in a measure to be split into unique and shared factors with other related measures. In this 
case, their analysis partialled out and controlled for the shared variance of self-esteem, body image, positive body-related selfjudgement, leaving only the unique variance from internalised weight stigma in a bi-dimensional measure.) on the Weight Bias Internalisation Scale. They then ran two mediation analyses: the first used the unidimensional version of internalised weight stigma, and the second used the bi-dimensional version. Their analysis found that the unidimensional version of internalised weight stigma mediated the relationship between experiences of stigma and eating behaviour outcomes (as described above), a finding consistent with other studies in this review. However, internalised weight stigma was a non-significant mediator of the target relationship when the scale was included in the model as a bi-dimensional measure (i.e., that had the shared variance of selfesteem and body image partitioned out and only included the unique variance that internalised weight stigma accounted for). This study suggests that the considerable overlap between internalised weight stigma, body image and self-esteem may be responsible for the mediation of experienced/perceived weight stigma and disordered eating relationship. This suggests greater conceptual clarity is needed regarding the nature and consequences of internalised weight stigma.

Depression and anxiety: Two studies measured anxiety and depression as outcomes. The findings were mixed for these studies. Specifically, only one study found a significant indirect effect of internalised weight stigma on the relationship between perceived weight stigma and depression and anxiety [33]. The other study found that internalised weight stigma did not mediate this relationship [43]. This may be for a number of reasons, given that although these samples had participants of a similar age, there were notable gender proportion and average BMI differences between samples. Moreover, though both samples were from clinical settings, the former study sampled outpatients from a clinical weight-loss programme, whereas the latter sampled a pre-bariatric surgery cohort-both of which may have inherent group differences. For example, some research suggests factors such as body image concerns are higher in those seeking bariatric surgery compared to those seeking less invasive treatment [5355]. The differences in the significance of findings, however, could not be explained by power as the samples were of similar size (see Table S5).

Other psychological outcomes: Five separate studies assessed body shame [31], body dissatisfaction [44], current exercise behaviour [34], healthcare experiences and behaviours [40], and emotional problems [45] as the outcome measure in their mediation analysis. The first three studies found that internalised weight stigma mediated the relationship between perceived/ experienced weight stigma and body shame, body dissatisfaction, and current exercise behaviour, respectively. The fourth study collected data across six countries and found that internalised weight stigma mediated the relationship between experienced weight stigma and eight different healthcare experiences and behaviours, such as perceived judgement by doctors about weight, general avoidance of the doctor, and perceived quality of healthcare received (full list of outcomes in Table S5). The last study found internalised weight stigma mediated the relationship between perceived/experienced weight stigma and emotional problems-but only for participants without overweight, and not for those with overweight.

Combined psychological outcomes: Two unpublished studies used latent variables as part of a structural equation model. These latent variables combined several constructs into a single outcome, and produced mixed results. The first study [42] combined disordered eating, body image, and depression to form one latent outcome measure. The authors found that internalised weight stigma mediated the relationship between experienced weight stigma and this outcome. The second study [48] measured a latent variable (psychological well-being) that consisted of a depression and a self-esteem measure. However, they found that internalisation of weight stigma did not mediate the relationship between experienced weight stigma and this outcome variable.

\section{Biological and social outcomes}

Bodily pain and parental weight talk: Lastly, two studies measured outcomes not classified as psychological: one study measured bodily pain (biological), the other study measured parental weight talk (social). Both studies found that internalised weight stigma mediated the relationship between perceived/ experienced weight stigma and bodily pain [29] and parental weight talk [50] (child-centred weight conversations, weight conversations about oneself, and weight conversations about others), respectively.

\section{DISCUSSION}

Weight stigma broadly refers to the social devaluation that individual's perceive/experience because of their weight [3]. Researchers have proposed and found evidence that internalised weight stigma mediates the relationship between experiences/ perceptions of weight stigma and negative health outcomes. Thus, the aim of the current study was to review the evidence on studies assessing internalised weight stigma as a mediator of the relationship between perceived/experienced weight stigma and biopsychosocial outcomes.

We identified 17 studies across 16 articles that met our inclusion criteria and were included in our systematic literature review. Almost all of these studies assessed psychological outcomes $(n=$ 15 ), and only $n=2$ assessed biological or social outcomes. Most studies in this review were of adequate quality. However, many reported insufficient statistical information about the effect size estimates of the indirect effect(s) and measures of error/dispersion for these estimates, instead only reporting significance. Moreover, almost all studies used the Weight Bias Internalisation Scale to measure internalised weight stigma. There is some research that shows there is limited evidence of content validity [56] for this measure. Thus, some caution should be exercised when interpreting these findings, as will be discussed below.

The findings in the current review provide support for the hypothesised pathways in the literature. Mainly, that internalised weight stigma mediates the relationship between experienced/ perceived weight stigma and adverse biopsychosocial outcomes [28] (Although these purported mechanisms suggest perceived/ experienced weight is a precursor for internalised weight stigma, this should not be taken to mean that it is the only precursor of internalisation). Specifically, these findings are consistent with the theoretical model proposed by Tylka et al. [28], in that internalised weight stigma mediated the relationship between perceived/ experienced weight stigma and psychological well-being and physical health. For psychological well-being, this included outcomes such as body shame, body dissatisfaction, healthcare experiences and behaviours, emotional problems, and parental weight talk (one study each). In addition, we found the mediating effect in 8 studies for disordered eating when the Weight Bias Internalisation Scale was used as a single-dimensional scale. Similar evidence was found for physical health as an outcome, where two studies in our review found that internalised weight stigma mediated the relationship between perceived/experienced weight stigma and current exercise behaviour and bodily pain, respectively. As mentioned above, however, the findings were mixed for depression and anxiety as outcome measures. With this exception in mind, the findings from most studies in our review are also consistent with other similar theoretical models in the 
literature [57, 58], which suggest that perceived/experienced weight stigma leads to greater internalised weight stigma, which in turn leads to decreased psychological well-being and poorer eating and weight-related behaviours.

Despite our inclusion criteria not being limited by design (studies could be cross-sectional, longitudinal, experimental), all studies included in our review were cross-sectional and correlational. Thus, although studies in this review provide necessary evidence about the purported mechanism, this evidence is not sufficient to be certain that internalised weight stigma plays a causal, mechanistic role in this relationship. The best approach to gather sufficient evidence would be to conduct experiments that (a) manipulate experience of weight stigma, (b) measure the subsequent effect of these experiences on internalisation and (c) identify changes in the outcome variables that come about after the internalisation of stigma. Designing studies that purposefully expose individuals to experiences of stigma, of course, poses an ethical problem. For this reason, it is imperative that future research investigates the causal pathways of this mechanism through longitudinal studies. For example, a longitudinal study could observe the temporal relationship between the experience of weight stigma and the following internalisation of weight stigma-which may then lead to negative outcomes. In the event of such studies finding evidence to support these models, the extent to which individuals' internalised weight stigma could then more confidently be a target construct in the assessment and treatment of both psychological and physical health.

\section{Limitations of the current literature}

Absence of theoretical models. Only four studies $[32,42,43,48]$ in the current review (two published, two unpublished) presented a theoretical model as the basis for evaluating the mechanism of interest. Most studies either (a) cited correlational research that suggested the mechanism might exist, or (b) cited a previous study that also investigated this mechanism for the same or similar outcome (e.g., disordered eating) as a rationale for their analysis. This, however, is to be expected, as most studies included in this review were exploratory in nature. Future research should aim to have a clear theoretical basis for testing their mediation models of interest (such as those presented above) $[28,57]$ to further this field of inquiry. In addition, no study in this review was pre-registered. To ensure transparent research in the future, all authors should pre-register their studies on platforms such as Open Science Framework [59].

Statistical reporting of mediation analyses. One of the main problems with the statistical reporting in the studies reviewed here was that the majority of studies reported insufficient statistical information. For example, 7 of 17 studies did not report any estimated effect size of the indirect effect. Of those that did report an indirect effect $(n=10)$, only Forbes and Donovan [31], Meadows and Higgs [41], and Puhl et al. [40] reported a standardised estimate effect size of their indirect effects (and measures of error/dispersion). In addition, 8 of 17 studies used structural equation modelling to assess their mediation, and although all authors using this method reported a measure of error for the overall model (root mean square error of approximation), none reported a measure of dispersion/error for each individual parameter estimate. These omissions do not allow readers to (a) appropriately understand the meaning of the reported indirect effect and compare the effect size across studies [60], (b) observe confidence interval width (or its proximity to zero) [61], or (c) observe levels of estimated precision in their reported coefficient (HB and XPG contacted all authors who used SEM for this information (e.g., indirect effect estimate, or measure of error) via email with only one response. The author from this study said they could not access the data due to the lockdowns from the COVID-19 pandemic.). In future, researchers should adhere to recommendations for mediation reporting (e.g., Cashin et al.) [38]. In addition, though we acknowledge the strict word/space limits journals place on authors, researchers should endeavour to include all relevant and recommended [62] information in future studies, and if journal word limits do not allow for this, to include this information in the supplementary material.

The inconsistencies in statistical reporting made it difficult to collate and compare the different findings. For this reason, we present a number of recommendations for reports of mediation analysis. When reporting indirect effects, it is essential that the parameter estimates for each component of the mediation are reported along with their standard error and/or confidence intervals [60]. In addition, a standardised measure of effect size would be useful to compare each measures' relative contributions-though this may not always be logical in cases of opposite values for the direct and indirect effects [60]. Wen and Fan [62] suggest researchers report several statistics. These include both the unstandardised (if meaningful) and standardised forms of the estimated effect sizes of (a) the total effect, (b) the indirect effect and (c) the direct effect, as well as their measures of error (i.e., standard error and confidence intervals).

Emphasis on psychological outcomes. As mentioned above, studies included in this review largely assessed psychological outcomes $(n=$ 15). In contrast, biological and social outcomes $(n=2)$ have been overlooked in this area, although there is both cross-sectional and experimental evidence that shows a link between experienced/ perceived weight stigma and some biological outcomes, such as cortisol levels $[63,64]$. Further, in the case of other minority groups (e.g., same-gender attracted individuals), there is evidence that internalised stigma mediates the relationship between expectations of discrimination and biological outcomes [65]. Thus, future research should investigate whether internalised weight stigma also mediates the relationship between experienced/perceived weight stigma and various physiological or biomarkers of health.

Internalised weight stigma as a unidimensional or a multidimensional construct. Notably, nearly all (16 of 17) studies in this review used the Weight Bias Internalisation Scale (referred to from here on as 'the scale') to measure internalised weight stigma in their mediation analysis. This is problematic for a few reasons. First, a recent systematic review of existing weight stigma measures [56] found that the scale (both original and follow-up validation/modification versions) did not assess content validity adequately (this was in fact the case for most existing weight stigma measures). According to COSMIN guidelines, when a measure does not meet the required content validity evidence checks, evaluation of the rest of the properties is not relevant [66]. Second, a recent study (which conducted an exploratory and confirmatory factor analysis on the original 19 items that the scale was created from) showed the data for the scale showed a poor fit for a unidimensional model, but a good to excellent fit onto a model with two subscales of weight-related distress (7 items) and weight-related self-devaluation (6 items), respectively [67]. Third, in a separate study, the same authors conducted a bi-factor analysis and found that, as a unidimensional construct, the scale does not distinguish itself sufficiently from related, established predictors of disordered eating (i.e., body image, self-esteem). The authors conclude by suggesting the findings of studies that have disordered eating as an outcome measure in the mechanism of interest are at least somewhat confounded by the related constructs of self-esteem and body image. This raises an important definitional issue about what internalised weight stigma is and the extent to which it is something other than poor body image or self-esteem. In sum, these three studies suggest that the frequent use of the Weight Bias Internalisation Scale throughout the literature to date may need to be revised.

In future, if researchers are planning on assessing internalised 
weight stigma, they could (a) aim to use other measures of internalisation that have demonstrated content validity as well as sound psychometric properties, (b) account for the shared variance in body image and self-esteem as related constructs, and (c) use the 6-item self-devaluation subscale from Meadows and Higgs [67] to measure internalised weight stigma. In addition to issues in the measurement of internalised weight stigma [68], the literature on weight stigma, in general, does not adequately make the distinction between perceived and experienced weight stigma. Thus, as a field of inquiry, we need to look closely at the conceptualisation and measurement of these differing constructs (see Papadopoulos et al. [56]).

\section{Strengths and limitations of the current review}

The current review provides the first synthesis of results for studies that assessed the mediating role of internalised weight stigma on the relationship between perceived/experienced weight stigma and any biopsychosocial outcomes. This review is both important and timely because the research in this area is in a preliminary stage. Thus, it is a good time to review the current evidence and consider it in light of our current understanding of the constructs to establish recommendations for future directions. Indeed, there are notable limitations with the available measures of weight stigma, particularly for internalised weight stigma (explained above) [56]. Improvements on measures of weight stigma will in turn allow the literature to move forward in a clear and efficient direction. Unfortunately, because of the lack of available data, we were unable to meta-analyse the results reported and provide an overview of the size of the effect(s) of internalised weight stigma as a mediator on the relationship between experienced/perceived weight stigma and negative biopsychosocial outcomes.

\section{Conclusions, implications and future directions}

In sum, we aimed to assess biopsychosocial outcomes, but found that most studies only assessed psychological outcomes. We also found consistent evidence for internalised weight stigma as a mediator of the relationship between perceived/experienced weight stigma and disordered eating. In addition, we found preliminary evidence for other outcomes in this mechanism, such as body shame, body dissatisfaction, current exercise behaviour, healthcare experiences and behaviours, emotional problems, bodily pain, and parental weight talk. However, the evidence was mixed for depression and anxiety as outcome measures (though there were a limited number of studies assessing these outcomes). Overall, the findings provide preliminary evidence that the relationship between perceived/experienced weight stigma and biopsychosocial outcomes-particularly psychological outcomes-is mediated by internalised weight stigma.

Given the abovementioned psychometric problems with the Weight Bias Internalisation Scale, there is scope for future researchers to further replicate these studies with a different measure of internalised weight stigma. In addition, Romano et al. [69] has found evidence that weight stigma concerns also mediates the relationship between perceived weight and overeating. Future research may want to consider including measures of perceived weight as a predictor of internalised weight stigma [70]. Future research should also conduct experimental (if possible and ethical) or longitudinal tests on the mediating role of internalised weight stigma on the relationship between perceived/experienced weight stigma and biopsychosocial outcomes. This would enable the evaluation of the causal pathways proposed in the theoretical models in the literature. Consequently, therapeutic and other health interventions targeted at improving physical and psychological outcomes can more confidently address the extent to which individuals internalise weight stigma.
Importantly, because weight stigma is pervasive and its sources are both individual (e.g., friends and family) and institutionalised (e.g., media messages, healthcare systems), trying to improve outcomes via change in the expressions of weight stigma is likely to take a long time. However, it might be possible to intervene on internalised weight stigma to prevent adverse health outcomes in the short term (i.e., while cultural changes are enacted to reduce weight stigma) (Importantly, our suggestion to address internalised weight stigma is in no way attempting to blame the victim of weight stigma. Instead, we are attempting to improve outcomes for the targets of weight stigma in the absence of societal and structural changes that eradicate weight stigma.). In a therapeutic context, for example, if a client presented with symptoms of an eating disorder, the clinician could attempt to reduce the negative outcomes associated with this condition by addressing the client's internalisation of weight stigma, through techniques such as cognitive restructuring and appraisal [71].

\section{REFERENCES}

1. Emmer C, Bosnjak M, Mata J. The association between weight stigma and mental health: a meta-analysis. Obes Rev. 2020;21:e12935.

2. Hunger JM, Dodd DR, Smith AR. Weight discrimination, anticipated weight stigma, and disordered eating. Eat Behav. 2020;37:101383.

3. Puhl R, Brownell KD. Bias, discrimination, and obesity. Obes Res. 2001;9:788-805.

4. Puhl RM, Heuer CA. The stigma of obesity: a review and update. Obesity. 2009;17:941.

5. Agerström J, Rooth D-O. The role of automatic obesity stereotypes in real hiring discrimination. J Appl Psychol. 2011;96:790.

6. Rubino F, Puhl RM, Cummings DE, Eckel RH, Ryan DH, Mechanick J, et al. Joint international consensus statement for ending stigma of obesity. Nat Med. 2020;26:485-97.

7. Myers $A$, Rosen JC. Obesity stigmatization and coping: relation to mental health symptoms, body image, and self-esteem. Int J Obes. 1999;23:221-30.

8. Hunger JM, Major B. Weight stigma mediates the association between BMI and self-reported health. Health Psychol. 2015;34:172.

9. Tomiyama AJ. Weight stigma is stressful. a review of evidence for the Cyclic Obesity/Weight-Based Stigma model. Appetite. 2014;82:8-15.

10. Daly $M$, Robinson $E$, Sutin AR. Does knowing hurt? Perceiving oneself as overweight predicts future physical health and well-being. Psychol Sci. 2017;28:872-81.

11. Papadopoulos S, Brennan L. Correlates of weight stigma in adults with overweight and obesity: a systematic literature review. Obesity. 2015;23:1743-60.

12. Jackson SE, Steptoe A, Beeken RJ, Croker H, Wardle J. Perceived weight discrimination in England: a population-based study of adults aged $\geqslant 50$ years. Int J Obes. 2015;39:858-64.

13. Wu YK, Berry DC. Impact of weight stigma on physiological and psychological health outcomes for overweight and obese adults: a systematic review. J Adv Nurs. 2018;74:1030-42.

14. Callahan D. Children, stigma, and obesity. JAMA Pediatr. 2013;167:791-2.

15. Latner JD, Puhl RM, Stunkard AJ. Cultural attitudes and biases toward obese persons. In: Akabas SR, Lederman SA, Moore BJ, (eds.) Textbook of obesity: biological, psychological and cultural influences. 1st ed, United Kingdom, UK: WileyBlackwell; 2012. p. 42-57.

16. Sutin AR, Stephan $Y$, Terracciano A. Weight discrimination and risk of mortality. Psychol Sci. 2015;26:1803-11.

17. Hunger JM, Major B, Blodorn A, Miller CT. Weighed down by stigma: how weightbased social identity threat contributes to weight gain and poor health. Soc Personal Psychol Compass. 2015;9:255-68.

18. Puhl R, Suh Y. Health consequences of weight stigma: implications for obesity prevention and treatment. Curr Obes Rep. 2015;4:182-90.

19. Vartanian LR, Porter AM. Weight stigma and eating behavior: a review of the literature. Appetite. 2016;102:3-14.

20. Almeida L, Savoy S, Boxer P. The role of weight stigmatization in cumulative risk for binge eating. J Clin Psychol. 2011;67:278-92.

21. Schvey NA, Puhl RM, Brownell KD. The impact of weight stigma on caloric consumption. Obesity. 2011;19:1957-62.

22. Major B, Hunger JM, Bunyan DP, Miller CT. The ironic effects of weight stigma. J Exp Soc Psychol. 2014;51:74-80.

23. Daly $M$, Sutin AR, Robinson E. Perceived weight discrimination mediates the prospective association between obesity and physiological dysregulation: evidence from a population-based cohort. Psychol Sci. 2019;30:1030-9. 
24. Lee KM, Hunger JM, Tomiyama AJ. Weight stigma and health behaviors: evidence from the Eating in America Study. Int J Obes. 2021;4:1499-509.

25. Major B, Rathbone JA, Blodorn A, Hunger JM. The countervailing effects of weight stigma on weight-loss motivation and perceived capacity for weight control. Pers Soc Psychol Bullet. 2020;46:1331-43.

26. Dixon JB. The effect of obesity on health outcomes. Mol Cell Endocrinol. 2010;316:104-8.

27. Durso LE, Latner JD. Understanding self-directed stigma: development of the weight bias internalization scale. Obesity. 2008;16:S80-S6.

28. Tylka TL, Annunziato RA, Burgard D, Daníelsdóttir S, Shuman E, Davis C, et al. The weight-inclusive versus weight-normative approach to health: evaluating the evidence for prioritizing well-being over weight loss. J Obes. 2014;2014:983495.

29. Olson KL, Mensinger JL. Weight-related stigma mediates the relationship between weight status and bodily pain: a conceptual model and call for further research. Body image. 2019;30:159-64.

30. Pötzsch A, Rudolph A, Schmidt R, Hilbert A. Two sides of weight bias in adolescent binge-eating disorder: adolescents' perceptions and maternal attitudes. Int J Eat Disord. 2018;51:1339-45.

31. Forbes $Y$, Donovan $C$. The role of internalised weight stigma and self-compassion in the psychological well-being of overweight and obese women. Aust Psychol. 2019;54:471-82

32. O'Brien KS, Latner JD, Puhl RM, Vartanian LR, Giles C, Griva K, et al. The relationship between weight stigma and eating behavior is explained by weight bias internalization and psychological distress. Appetite. 2016;102:70-6.

33. Magallares A, Bolaños-Rios P, Ruiz-Prieto I, de Valle PB, Irles JA, Jáuregui-Lobera I. The mediational effect of weight self-stigma in the relationship between blatant and subtle discrimination and depression and anxiety. Span J Psychol. 2017;20: e4, 1-7.

34. Pearl RL, Puhl RM, Dovidio JF. Differential effects of weight bias experiences and internalization on exercise among women with overweight and obesity. J Health Psychol. 2015;20:1626-32.

35. Moher D, Shamseer L, Clarke M, Ghersi D, Liberati A, Petticrew M, et al. Preferred reporting items for systematic review and meta-analysis protocols (PRISMA-P) 2015 statement. Syst Rev. 2015;4:1.

36. Shea BJ, Reeves BC, Wells G, Thuku M, Hamel C, Moran J, et al. AMSTAR 2: a critical appraisal tool for systematic reviews that include randomised or nonrandomised studies of healthcare interventions, or both. BMJ. 2017;358:j4008.

37. Ouzzani M, Hammady H, Fedorowicz Z, Elmagarmid A. Rayyan-a web and mobile app for systematic reviews. Syst Rev. 2016;5:210.

38. Cashin A, McAuley J, Lamb SE, Hopewell S, Kamper S, Williams C, et al. Items for consideration in a reporting guideline for mediation analyses: a Delphi study. 2020;26:106

39. Hayes AF. Introduction to mediation, moderation, and conditional process analysis: a regression-based approach. Guilford publications; 2017.

40. Puhl RM, Lessard LM, Himmelstein MS, Foster GD. The roles of experienced and internalized weight stigma in healthcare experiences: Perspectives of adults engaged in weight management across six countries. PLoS ONE. 2021;16: e0251566.

41. Meadows A, Higgs S. A bifactor analysis of the Weight Bias Internalization Scale: What are we really measuring? Body Image. 2020;33:137-51.

42. Oehlhof MEW. Self-objectification among overweight and obese women: an application of structural equation modeling. Bowling Green State University; 2011.

43. Braun TD, Quinn DM, Stone A, Gorin AA, Ferrand J, Puhl RM, et al. Weight bias, shame, and self-compassion: risk/protective mechanisms of depression and anxiety in prebariatic surgery patients. Obesity. 2020;28:1974-83.

44. Romano KA, Heron KE, Henson JM. Examining associations among weight stigma, weight bias internalization, body dissatisfaction, and eating disorder symptoms: does weight status matter? Body Image. 2021;37:38-49.

45. Zuba $A$, Warschburger $P$. The role of weight teasing and weight bias internalization in psychological functioning: a prospective study among school-aged children. Eur Child Adolesc Psychiatry. 2017;26:1245-55.

46. Thompson JK, Cattarin J, Fowler B, Fisher E. The perception of teasing scale (POTS): a revision and extension of the physical appearance related teasing scale (PARTS). J Pers Assess. 1995;65:146-57.

47. Remmert JE, Convertino AD, Roberts SR, Godfrey KM, Butryn ML. Stigmatizing weight experiences in health care: Associations with BMI and eating behaviours. Obes Sci Pract. 2019;5:555-63.

48. Elison ZM. Psychological well-being in overweight adults: the effects of percieved weight discrimination, internalized weight stigma, and coping. Purdue University; 2017.

49. Durso LE, Latner JD, Hayashi K. Perceived discrimination is associated with binge eating in a community sample of non-overweight, overweight, and obese adults. Obes Facts. 2012;5:869-80.
50. Pudney EV, Himmelstein MS, Puhl RM. The role of weight stigma in parental weight talk. Pediatr Obes. 2019;14:e12534.

51. Pearl RL, Puhl RM. Measuring internalized weight attitudes across body weight categories: validation of the modified weight bias internalization scale. Body Image. 2014;11:89-92.

52. Lillis J, Luoma JB, Levin ME, Hayes SC. Measuring weight self-stigma: the weight self-stigma questionnaire. Obesity. 2010;18:971-6.

53. Sarwer DB, Polonsky HM. Body image and body contouring procedures. Aesthet Surg J. 2016;36:1039-47.

54. Fischer L, Wekerle A-L, Sander J, Nickel F, Billeter AT, Zech U, et al. Is there a reason why obese patients choose either conservative treatment or surgery? Obes Surg. 2017;27:1684-90.

55. Makarawung D, Monpellier V, van den Brink F, Woertman L, Zijlstra H, Mink van der Molen A, et al. Body image as a potential motivator for bariatric surgery: a case-control study. Obes Surg. 2020;30:3768-75.

56. Papadopoulos S, de la Piedad Garcia X, Brennan L. Evaluation of the psychometric properties of self-reported weight stigma measures: a systematic literature review. Obes Rev. 2021;22:e13267.

57. Lee MS, Gonzalez BD, Small BJ, Thompson JK. Internalized weight bias and psychological wellbeing: an exploratory investigation of a preliminary model. PLOS ONE. 2019;14:e0216324.

58. Ratcliffe D, Ellison N. Obesity and internalized weight stigma: a formulation model for an emerging psychological problem. Behav Cog Psychother. 2015;43:239.

59. Foster ED, Deardorff A. Open science framework (OSF). J Med Libr Assoc: JMLA. 2017;105:203.

60. Preacher KJ, Kelley K. Effect size measures for mediation models: quantitative strategies for communicating indirect effects. Psychol Methods. 2011;16:93.

61. Götz M, O’Boyle EH, Gonzalez-Mulé E, Banks GC, Bollmann SS. The “Goldilocks Zone":(Too) many confidence intervals in tests of mediation just exclude zero. Psychol Bull. 2020;147:95-114

62. Wen Z, Fan X. Monotonicity of effect sizes: Questioning kappa-squared as mediation effect size measure. Psychol Methods. 2015;20:193.

63. Tomiyama AJ, Epel ES, McClatchey TM, Poelke G, Kemeny ME, McCoy SK, et al. Associations of weight stigma with cortisol and oxidative stress independent of adiposity. Health Psychol. 2014;33:862.

64. Schvey NA, Puhl RM, Brownell KD. The stress of stigma: exploring the effect of weight stigma on cortisol reactivity. Psychosom Med. 2014;76:156-62.

65. Norcini Pala A, Hart RP, Steca P. Minority stress, depression and HIV-progression biomarkers: an exploratory study on a sample of Italian HIV-positive gay and bisexual men. J Gay Lesbian Ment Health. 2015;19:244-60.

66. Terwee $C B$, Prinsen $C$, Chiarotto A, De Vet H, Bouter LM, Alonso J, et al. COSMIN methodology for assessing the content validity of PROMs-user manual. Amsterdam: VU University Medical Center; 2018.

67. Meadows A, Higgs S. The multifaceted nature of weight-related self-stigma: validation of the two-factor weight bias internalization scale (WBIS-2F). Front Psychol. 2019;10:808.

68. Austen E, Pearl RL, Griffiths S. Inconsistencies in the conceptualisation and operationalisation of internalized weight stigma: a potential way forward. Body Image. 2020;36:3-5.

69. Romano E, Haynes A, Robinson E. Weight perception, weight stigma concerns, and overeating. Obesity. 2018;26:1365-71.

70. Robinson E, Haynes A, Sutin A, Daly M. Self-perception of overweight and obesity: a review of mental and physical health outcomes. Obes Sci Pract. 2020;6:552-61.

71. Pearl RL, Hopkins CH, Berkowitz Rl, Wadden TA. Group cognitive-behavioral treatment for internalized weight stigma: a pilot study. Eat Weight Disord. 2018;23:357-62.

\section{AUTHOR CONTRIBUTIONS}

Conceptualisation, systematic search, and double screening: XPG and HB. Data extraction: HB. XPG and HB wrote the manuscript; LB and LK edited the manuscript. All authors read and approved the final manuscript.

\section{FUNDING}

HB is supported by an Australian Government Research Training Program (RTP) scholarship through ACU.

\section{COMPETING INTERESTS}

The authors declare no competing interests. 


\section{ADDITIONAL INFORMATION}

Supplementary information The online version contains supplementary materia available at https://doi.org/10.1038/s41366-021-00982-4.

Correspondence and requests for materials should be addressed to Xochitldela Piedad Garcia.
Reprints and permission information is available at http://www.nature.com/ reprints

Publisher's note Springer Nature remains neutral with regard to jurisdictional claims in published maps and institutional affiliations. 